\title{
New Cancer Drug Approvals From the Perspective of a Universal Healthcare System: Analyses of the Pan-Canadian Oncology Drug Review Recommendations
}

\author{
Saroj Niraula, MBBS, MD, MSc, ${ }^{\mathrm{a}, \mathrm{b}}$ and Zoann Nugent, $\mathrm{PhD} \mathrm{D}^{\mathrm{a}, \mathrm{b}}$
}

\begin{abstract}
Background: FDA approvals do not consider cost, but they set the tone for regulatory approvals worldwide, including in countries with universal healthcare where cost-effectiveness, utility, and adoption feasibility are considered rigorously. Methods: Data from the pan-Canadian Oncology Drug Review (pCODR), a national drug review system that makes evidence-based funding recommendations to Canada's provinces and territories, were collected. Our objectives were to assess (1) temporal trends in cost and efficacy of drugs reviewed, (2) correlations among magnitude of benefits, cost, and pCODR decisions, and (3) predictors of approvals. Results: A total of 60 drugs for 91 indications were reviewed by pCODR from January 2012 to January 2018. Of the 91 reviews (approved previously by FDA), 18 received negative recommendations on the grounds of inadequate clinical benefits; $87 \%(64 / 73)$ of those approved were conditional on improvement in cost. Surrogate outcomes were used to support approvals in $83 \%$ of the reviews, which were not correlated with overall survival $\left(r_{\text {spearman }}=\right.$ +0.16 ; $P=.24$ ). Median cost/quality-adjusted life years (QALY) increased by $36 \%$ per annum (quantile regression, $P=.0029$ ), although benefits in overall and progression-free survival were stable ( $P=.21$ and .65 , respectively). Median-based incremental cost-effectiveness ratio (ICER) of new drugs was $\$ 186,403$ CAD (range, $\$ 7,200$ to $\$ 2.1$ million). Higher ICER was a strong predictor of a negative pCODR recommendation $(P<.01)$. Conclusions: A substantial number of cancer drugs that are FDA approved for public use do not meet Canadian standards for efficacy. Cost of cancer drugs increases by a third annually in Canada, but the benefits-measured mostly with surrogates that did not correlate with survival-are stable. With finite resources to share among multiple societal priorities, such as education and preventive health, cancer drug cost may be unsustainable despite price regulation.
\end{abstract}

J Natl Compr Canc Netw 2018;16(12):1460-1466 doi: 10.6004 ljnccn.2018.7084

Therapeutic index, defined as the ratio of benefits to harms, is the primary consideration before the FDA approves a new healthcare intervention for public use. It is illegal for the FDA to consider drug costs prior to approvals. Nevertheless, these approvals have direct impact on the dynamics of the global health economy, because they exert immediate pressure on the market worldwide to bring interventions to patients. Global cost of cancer drugs currently exceeds US $\$ 100$ billion and is expected to surpass $\$ 150$ billion by $2020 .{ }^{1}$ Unfortunately, unlike most other commodities, the cost of new cancer interventions is driven by affordability within the market, and

aCancerCare Manitoba, and bUniversity of Manitoba, Winnipeg, Manitoba, Canada.

Submitted June 27, 2018; accepted for publication September 12, 2018.

Dr. Niraula has disclosed that he has received grant/research support

from Pfizer. Dr. Nugent has disclosed that she has no financial interests, not necessarily by added value of the intervention. ${ }^{2,3}$ The unprecedented spending in cancer care includes countries that actively regulate this cost. ${ }^{4}$

High drug prices are most consequential to individual patients in countries with inadequate insurance coverage, such as the United States, and in developing countries. In many European countries and Canada, where healthcare is funded publicly, the economic burden shifts more toward the government. These countries actively negotiate the prices of new healthcare interventions. In Canada, since 2011, newly approved cancer drugs are reviewed by an independent national

arrangements, affiliations, or commercial interests with the manufacturers of any products discussed in this article or their competitors.

Correspondence: Saroj Niraula, MBBS, MD, MSc, University of Manitoba, Room L1-101-13, 409 Tache Avenue, Winnipeg, Manitoba R2H 2A6,

Canada. Email: sniraula@cancercare.mb.ca 
process called the pan-Canadian Oncology Drug Review (pCODR), a branch of Canadian Agency for Drugs and Technologies in Health (CADTH). With the stated objective "to bring consistency and clarity to the assessment of cancer drugs," pCODR provides funding recommendations to Canada's provinces and territories based on review of evidence with regard to efficacy, toxicity, and cost using the deliberative framework for drug reimbursement. ${ }^{5}$ This framework is built on 4 pillars: clinical benefit, economic evaluation, patient-based values, and adoption feasibility. pCODR recommendations can be broadly divided into positive (ie, approved) versus negative, and conditional versus full approvals. Therefore, the strategy is for pCODR to function as a watchdog to keep expensive drugs with little value out of the market. Lapatinib in metastatic breast cancer and ofatumumab in chronic lymphocytic leukemia are examples of drugs that were approved by the FDA but received a negative $\mathrm{pCODR}$ recommendation because of low added value.

Previous studies have looked at methodological issues and consistencies in economic evaluations by pCODR. ${ }^{6,7}$ Almost all services related to cancer treatment are publicly funded in Canada, and cancer drugs are actively price-negotiated. This poses unique challenges and opportunities, with drug approvals that could vary systematically from private healthcare systems. Therefore, we analyzed the trends of approvals in terms of magnitude of benefits and costs of cancer drugs reviewed by $\mathrm{pCODR}$ since its establishment in 2011, and assessed predictors of pCODR approvals.

\section{Methods}

\section{Search Criteria}

We reviewed the CADTH website to identify all cancer drugs reviewed by pCODR from its inception to January $31,2018 .^{5}$ We then reviewed the pCODR final recommendation documents to extract data on efficacy, cost, and cost-effectiveness for each review. All drugs used for treatment of solid tumors and hematologic malignancies were included. We checked references provided in the pCODR documents to access the primary publications of pivotal clinical trials used to support recommendations and verified respective efficacy data. For resubmissions, final pCODR documents were used.

\section{Data Extraction}

Data were collected in prespecified forms by the first author (S.N.), and the collected data were reviewed against original documents by the second author (Z.N.). Any discrepancies identified were resolved by consensus between the authors. Data fields included information on drugs under review and respective indication, pCODR recommendations, rationale for pCODR recommendation and any conditions thereof, efficacy results reported in the pivotal clinical trials of respective drugs, end points used, and costeffectiveness data from pCODR economic analysis. The FDA website was accessed to check the status of drugs in the United States and their approval date in order to compare timelines of approvals between the FDA and pCODR. ${ }^{8}$

\section{Temporal Trends}

The expectation is that in countries with universal healthcare, drug prices are well controlled. To test this, we calculated the temporal trends in (1) magnitude of benefits reported in pivotal clinical trials used to support the drug reviews, and (2) cost/quality-adjusted life years (QALY) of the reviewed drugs. Drug costs were taken from the list price submitted to pCODR for cost-effectiveness analyses and are in Canadian dollars (valued currently at approximately $0.75 \times$ USD). List price is the price submitted by the manufacturer to pCODR; this is the price before negotiations. Prices after negotiations are confidential. Cost data were not normally distributed, and therefore are not amenable to linear regression analyses. However, their median values could be modeled, and therefore we used quantile regression modeling to assess their temporal trends. Because only 2 drugs were reviewed in 2018, their data were combined with the 2017 observations for the purpose of these analyses.

\section{Differences in Drugs With Positive Versus Negative pCODR Recommendations}

To assess if key cost and efficacy variables are associated with type of $\mathrm{pCODR}$ recommendation, we used Wilcoxon 2-sample test to explore the differences between drugs that received positive versus negative recommendations based on hazard ratio (HR) for overall survival (OS), HR for progression-free survival (PFS), monthly costs, cost/QALY, and incremental cost-effectiveness ratio (ICER). Whenever 
cost was presented as a range, we used the median for analysis.

\section{Correlations}

For drugs that reported both PFS and OS, we used Spearman correlation analysis to calculate the correlation between these end points. Similarly, we calculated the correlation between the HR of both PFS and OS of all reviewed drugs to their respective cost/ QALY and ICER.

\section{Predictors of Approval}

To assess variables predicting positive pCODR recommendations, we performed a linear regression analysis with ICER, HR-OS, and HR-PFS as dependent variables and pCODR recommendations (assessed as not approved, conditionally approved, or unconditionally approved) as predictors.

\section{Results}

From inception to January 31, 2018, 60 new cancer drugs for 91 indications were reviewed by pCODR (see supplemental eTable 1, available with this article at JNCCN.org). Final recommendations by pCODR were provided after a median of 15 months (range, 2-136 months) following FDA approvals. Of the drugs reviewed, 39 were in the first-line setting, 41 in second-line, and 11 in either beyond second-line or as maintenance. Of the 91 reviews, which were all previously approved by the FDA, 18 received a negative $\mathrm{pCODR}$ recommendation. Uncertain and/or inadequate net clinical benefits were stated as the reasons for the negative recommendation on all of these drugs. Of the 73 that received a positive pCODR recommendation, 87\% (64/73) were approved conditional on improvement in costeffectiveness to an acceptable level. Of the 91 reviews, 28 were supported by clinical trials with OS as the primary or coprimary end point. The remaining studies included PFS, event-free survival, or response rates as the primary end point. Interestingly, 3 drugs-pazopanib for renal cell carcinoma and ceritinib and crizotinib for non-small cell lung cancer-were reviewed based on improvement in PFS but actually had a shorter OS in the experimental arm compared with the control arm by 1,2 , and 2.5 months, respectively.

\section{Monthly Costs, Cost/QALY, and ICER}

Median monthly cost of new drugs reviewed by pCODR was $\$ 8,213$ CAD (range, $\$ 1,016-\$ 97,440$ ), and median cost per QALY of the new drugs was $\$ 75,957$ (range, $\$ 5,074-\$ 522,810$ ). There was only 1 drug, aldesleukin for treatment of in-transit melanoma, with a negative cost/QALY (of $-\$ 5,074$ ); however, this analysis was driven by the costs of interleukin-2, alternate systemic therapies (ipilimum$\mathrm{ab}$ and vemurafenib), isolated limb infusion, and radiation. Median ICER of new drugs compared with controls was $\$ 186,403$ (range, $\$ 7,265-\$ 2,143,880$ ) (supplemental eTable 1).

\section{Temporal Trends}

The magnitude of benefits offered by the new drugs as assessed by HR-PFS and HR-OS did not show any statistical trend over time (quantile regression, $P=.21$ and .65 , respectively). However, there was a statistically significant increment in median cost/ QALY by $36 \%$ per annum $(P=.0029)$. A sensitivity analysis including only the approved drugs continued to show significant increase in cost/QALY at $26 \%$ per annum $(P=.014)$. Monthly drug costs increased significantly $(P=.019)$ by $11 \%$ per annum. Disconnect between change in efficacy and cost highlights the need for incorporating value in drug pricing. Similarly, another measure of value, ICER did not increase significantly over the study period $(P=.16)$ (Figure 1).

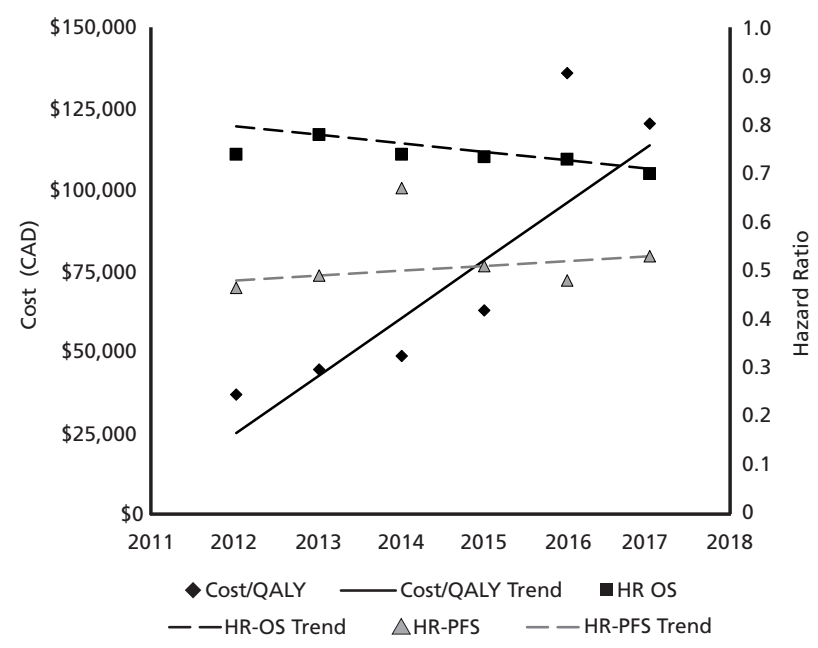

Figure 1. Comparative representation of change in efficacy and cost of new anticancer drugs reviewed by the pan-Canadian Oncology Drug Review from 2012 to 2018.

Abbreviations: HR, hazard ratio; OS, overall survival; PFS, progression-free survival; QALY, quality-adjusted life years. 


\section{Correlations}

HR-OS and HR-PFS were not significantly correlated overall $\left(\mathrm{r}_{\text {Spearman }}=+0.16 ; P=.24\right)$ or separately among drugs that received positive $\left(\mathrm{r}_{\text {Spearman }}=+0.15\right.$; $P=.29)$ or negative recommendations $\left(r_{\text {Spearman }}=\right.$ $-0.006 ; P=.99)$. HR-PFS achieved borderline statistical significance, with negative correlation to cost/ QALY, meaning that higher cost was associated with better PFS $\left(\mathrm{r}_{\text {Spearman }}=-0.261 ; P=.04\right)$. The pattern was also found (without formal significance in the smaller sample) among the approved drugs $\left(\mathrm{r}_{\text {Spearman }}=\right.$ $-0.251 ; P=.08)$, but not among those that were unapproved $\left(\mathrm{r}_{\text {Spearman }}=-0.109 ; P=.76\right)($ Table 1$)$.

\section{Differences Between Drugs With Positive and Negative Recommendations}

No differences reached formal significance when drugs that received positive recommendations were compared with those that received negative recommendations based on PFS (Wilcoxon, $P=.14$ ), cost/ month in the experimental arm (Wilcoxon, $P=.39$ ), cost/QALY (Wilcoxon, $P=.31$ ), and ICER (Wilcoxon, $P=.11)$. A nonsignificant trend was observed for approved drugs that had better OS compared with those not approved (Wilcoxon, $P=.06$ ) (Table 2).

\section{Predictors of Approval}

Of the 91 reviews, 10 did not provide cost-effectiveness data. Absence of cost-effectiveness data had a strong association with negative pCODR recommendation $(P<.001)$. Tertile regression demonstrat-

\begin{tabular}{|c|c|c|c|c|}
\hline Term 1 & Term 2 & $\mathbf{N}$ & $r_{\text {Spearman }}$ & $P$ Value \\
\hline \multicolumn{5}{|l|}{ All drugs } \\
\hline \multirow[t]{2}{*}{ HR-OS } & Cost/QALY & 54 & -0.207 & .13 \\
\hline & ICER & 53 & +0.080 & .57 \\
\hline \multirow[t]{2}{*}{ HR-PFS } & Cost/QALY & 61 & -0.261 & .04 \\
\hline & ICER & 61 & +0.044 & .74 \\
\hline \multicolumn{5}{|c|}{ Approved drugs } \\
\hline \multirow[t]{2}{*}{ HR-OS } & Cost/QALY & 45 & -0.058 & .71 \\
\hline & ICER & 46 & +0.107 & .48 \\
\hline \multirow[t]{2}{*}{ HR-PFS } & Cost/QALY & 51 & -0.251 & .08 \\
\hline & ICER & 53 & -0.010 & .94 \\
\hline \multicolumn{5}{|c|}{ Not approved drugs } \\
\hline \multirow[t]{2}{*}{ HR-OS } & Cost/QALY & 9 & -0.251 & .51 \\
\hline & ICER & 7 & -0.450 & .31 \\
\hline \multirow[t]{2}{*}{ HR-PFS } & Cost/QALY & 10 & -0.109 & .76 \\
\hline & ICER & 8 & +0.323 & .43 \\
\hline
\end{tabular}

Abbreviations: HR, hazard ratio; ICER, incremental cost-effectiveness ratio; QALY, quality-adjusted life years; OS, overall survival; PFS, progression-free survival. ed that increment in ICER was a negative predictor of approval by pCODR $(P=.0083)$. Increment in HR-OS or HR-PFS did not predict for pCODR recommendation ( $P=.16$ and .051 , respectively) (Table 3). Sparse data did not allow for a multivariable adjustment in this analysis, and therefore our results should not be considered definitive.

\section{Discussion}

Despite relatively modest, stable improvements in efficacy, measured mostly with surrogate outcomes that were not correlated with OS in the current analysis, the cost/QALY of new anticancer drugs increased by more than a third each year, and monthly drug costs increased by $11 \%$ per year since the inception of pCODR in 2011, posing a major challenge for continued cost containment. We also highlight the discrepancy between FDA approvals and Canadian funding recommendations, with almost a quarter of FDA-approved drugs not meeting Canadian standards for efficacy, and most of those approved deemed cost-ineffective at the list price, although the final negotiated price is kept confidential. ${ }^{9}$

Steep increases in cancer drug prices have been at the center of cancer policy debates, mainly in the second decade of the century, and although multiple avenues of interventions have been identified, they have mostly been futile thus far. ${ }^{10-12}$ Countries with universal healthcare systems, such as Canada and the United Kingdom, have adopted systems to regulate funding for drugs based on cost-effectiveness, with involvement of agencies such as pCODR and the National Institute for Health and Care Excellence (NICE), respectively. Restriction of access to expensive drugs in this way may reduce healthcare spending overall, but this does not decrease drug prices directly. Moreover, this may delay access to and deprive patients of potentially useful but costineffective treatments. Furthermore, such a strategy may invite a risk of misusing "alternate government pockets" to pay for expensive drugs. For example, cancer drug funds-pools of money provisioned for innovative treatments-were once used to pay for drugs deemed cost-ineffective by NICE. This practice ceased only due to significant criticism. ${ }^{13}$

Most pCODR approvals (87\%) were conditional based on improvement in cost-effectiveness to an acceptable level. Although such assessment may help 
Niraula and Nugent

Table 2. Differences Between Drugs With Positive and Negative Recommendations

\begin{tabular}{|c|c|c|c|c|c|c|}
\hline Variable & pCODR Recommendation & $\mathbf{N}$ & Median & Lower Quartile & Upper Quartile & $\begin{array}{c}\text { Wilcoxon 2-Sample Test } \\
\text { Probability }\end{array}$ \\
\hline \multirow[t]{2}{*}{ HR-OS } & Negative & 10 & 0.80 & 0.74 & 0.86 & 0.06 \\
\hline & Positive & 51 & 0.72 & 0.66 & 0.79 & \\
\hline \multirow[t]{2}{*}{ HR-PFS } & Negative & 12 & 0.63 & 0.52 & 0.71 & 0.14 \\
\hline & Positive & 58 & 0.49 & 0.39 & 0.65 & \\
\hline \multirow[t]{2}{*}{ Cost/Month: experimental arm (CAD) } & Negative & 18 & $\$ 6,466$ & $\$ 4,453$ & $\$ 10,409$ & 0.39 \\
\hline & Positive & 67 & $\$ 8,213$ & $\$ 5,305$ & $\$ 11,479$ & \\
\hline \multirow[t]{2}{*}{ Cost/Month: control arm (CAD) } & Negative & 10 & $\$ 5,589$ & $\$ 2,611$ & $\$ 10,000$ & 0.08 \\
\hline & Positive & 48 & $\$ 2,938$ & $\$ 639$ & $\$ 6,037$ & \\
\hline \multirow[t]{2}{*}{ Cost/QALY (CAD) } & Negative & 15 & $\$ 40,542$ & $\$ 29,373$ & $\$ 154,775$ & 0.31 \\
\hline & Positive & 66 & $\$ 75,209$ & $\$ 43,768$ & $\$ 127,187$ & \\
\hline \multirow[t]{2}{*}{ ICER (CAD) } & Negative & 13 & $\$ 204,047$ & $\$ 181,345$ & $\$ 363,719$ & 0.11 \\
\hline & Positive & 68 & $\$ 180,801$ & $\$ 132,584$ & $\$ 271,216$ & \\
\hline
\end{tabular}

Abbreviations: HR, hazard ratio; ICER, incremental cost-effectiveness ratio; OS, overall survival; pCODR, pan-Canadian Oncology Drug Review; PFS, progression-free survival; QALY, quality-adjusted life years.

in future negotiation of prices with manufacturers before securing public funding, the extent of improvement expected of a drug to reach an acceptable costeffectiveness threshold remains unaddressed, and therefore this is left in the remit of negotiators, with potential for subjective variability. Assigning a minimum acceptable improvement in drug cost (based on efficacy and tolerability, for example) would help set standards and minimize heterogeneity in drug prices. It was reassuring to note in our analysis that an increase in ICER was a strong negative predictor of a pCODR recommendation. Again, setting an ICER threshold for approval based on indications may allow for a more transparent and homogeneous approval process.

Although higher ICER was a predictor of negative pCODR recommendation, which is concordant with a previous analysis suggesting that clinical aspects (rather than cost) carries more weight in $\mathrm{pCO}$ DR decisions, ${ }^{7}$ it was interesting to note that neither of the effectiveness measures (HR-OS and HR-PFS) had significant association with a positive $\mathrm{pCODR}$ recommendation. Similarly, none of the other cost

\begin{tabular}{|c|c|c|c|c|c|}
\hline Single Predictor & $\mathrm{N}$ & $\begin{array}{l}\text { Parameter } \\
\text { Estimate }\end{array}$ & SE & $t$ Value & $P$ Value \\
\hline ICER tertiles & 81 & -0.19 & 0.07 & -2.71 & .0083 \\
\hline Tertiles of HR-OS & 61 & -0.12 & 0.08 & -1.44 & .16 \\
\hline Tertiles of HR-PFS & 70 & -0.15 & 0.07 & -1.99 & .051 \\
\hline
\end{tabular}

Abbreviations: HR, hazard ratio; ICER, incremental cost-effectiveness ratio; OS, overall survival; pCODR, pan-Canadian Oncology Drug Review; PFS, progressionfree survival. and/or effectiveness variables were associated statistically with pCODR decisions. This is rather counterintuitive in the Canadian context, wherein committees like pCODR are tasked explicitly to incorporate cost-effectiveness in the process; subjective factors such as patient values may alter the balance between access to treatment and potential benefits and cost. Furthermore, acceptable efficacy can differ based on disease-specific variables, such availability of alternate treatments and rarity of the tumor.

The focus of regulatory authorities, such as the FDA and the European Medicines Agency, is primarily to grant timely access to safe, effective, and high-quality drugs, but these factors are independent of price. The tendency is to approve drugs meeting a certain threshold for efficacy and to trust clinicians with ground-level decisions on actual use of drugs after approvals. A common understanding of regulatory approvals among physicians may be different from the expectations of the FDA. Clinicians are known to have a poor understanding of drug costs, and have a tendency to underestimate the price of expensive drugs and overestimate the price of inexpensive ones. ${ }^{14}$ Furthermore, important guidelines that clinicians rely heavily on, such as those issued by NCCN, tend to list drugs in the compendium for some indications regardless of FDA approval status or cost. ${ }^{15}$ These factors together increase the potential for inappropriate overuse of costly drugs with marginal benefits. Oversight of drug use, such as by institutional drug formulary committees, can protect against this practice at the final stage of drug utilization. 
In the United States, initiatives such as the Institute for Clinical and Economic Review seek to provide a value-based price benchmark for drugs across multiple disciplines based on the efficacy, incremental benefits, extra costs or savings, and overall cost of treating the entire population. Strengthening the negotiating power of these initiatives and providing them with authority to implement health policies may promote a fairer value-based drug market, although the fragmented system of payers is likely to require major commitment at the federal level to overcome challenges in implementing such policies. In addition, the cost-effectiveness of cancer drugs in the United States is worse than in Canada; for instance, the drug palbociclib used in metastatic breast cancer has a list price in Canada of $\$ 6,250$ CAD as opposed to a wholesale price in the United States of $\$ 13,550$ USD. ${ }^{16,17}$ Therefore, strategies that need to be adopted for drug regulations will vary by country, in addition to conventional variables, such as prevalence of the indication and status of alternative treatment options.

Our study had some limitations. First, as concluded by previous studies, there are methodological issues in the studies presented to pCODR to support review, such as quality of clinical data, uncertainties with indirect comparisons, time horizons considered, and choice of utility estimates. ${ }^{6}$ Second, we used new oncology drugs and oncology drugs with new indications in the 6-year period since the inception of pCODR in 2011. Although this is a short period, we believe this is a reasonable representation of recent trend in drug approvals. Third, we adopted results from cost-effective analyses performed by pCODR, and therefore any assumptions necessary to conduct such analyses vary among the drugs and clinical indication, although well-accepted methodologies are used by pCODR for these analyses. Similar analyses from other public healthcare systems will help validate our results. Fourth, variables such as detailed quality measures and strength of supporting studies that could potentially predict pCODR outcomes would not be easily analyzed.

The costs and cost-effectiveness analyses used in our study are based on outcomes of patients from controlled settings of clinical trials. Newly approved anticancer drugs lead to higher toxicity and more treatment discontinuations compared with controls, even in the controlled setting of clinical trials. ${ }^{18}$ After approval, when the drugs are used by an unselected general population, the likelihood of toxicities will be higher than in clinical trials, which in turn will lead to a further increase in overall cost of treatment. ${ }^{19}$ Similarly, inability to complete treatments due to toxicity (treatment discontinuations before progression) will lead to higher chances of cancer progression and hence greater cost of treatment. The incremental cost presented in our analysis therefore represents only a fraction of the overall increment in cost of cancer care. Nevertheless, spending on drugs in oncology is the highest among any medical specialty and is approaching unsustainable levels. ${ }^{20,21}$ Controlling these costs is therefore a necessary step toward overall improvement in sustainability of cancer care.

\section{Conclusions}

Despite a relatively stable increment in efficacy compared with the previous standard of care, costs of cancer drugs have been increasing by a third annually in the "price-regulated" cancer drug market of Canada. This figure calls for emergency action because this trend is unsustainable even in the wealthiest of nations. It is prudent to break the historic trend of implementing interventions following, rather than preceding, an impending health tragedy ${ }^{22,23}$; immediate regulatory measures to control unjustified expenditure in cancer drugs may prevent possible compromises in other areas of important societal priorities, such as education and preventive health. Countries with universal healthcare systems, such as Canada, have the ability to set a precedent for the rest of the world in this regard. Collaborative efforts among national policymakers, the pharmaceutical industry, opinion leaders, patient advocacy groups, and regulatory agencies are imperative for the continued containment of the cost of cancer care.

\section{References}

1. Berkrot B. Global cancer drug spending to exceed $\$ 150$ billion by 2020 IMS report. Reuters. June 2, 2016. Available at: https://www.reuters.com/ article/us-health-cancer-spending/global-cancer-drug-spending-to-exceed-
150-billion-by-2020-ims-report-idUSKCNOYOOBQ. Accessed March 18, 2018.

2. Bach PB. New math on drug cost-effectiveness. $N$ Engl J Med 2015;373:1797-1799. 
3. Prasad V, Mailankody S. Research and development spending to bring a single cancer drug to market and revenues after approval. JAMA Intern Med 2017;177:1569-1575.

4. de Oliveira C, Weir S, Rangrej J, et al. The economic burden of cancer care in Canada: a population-based cost study. CMAJ Open 2018;6:E1-10.

5. CADTH/pCODR. pCODR Expert Review Committee Deliberative Framework. Published March 2011. Available at: https://www.cadth.ca/ sites/default/files/pcodr/pCODR\%27s\%20Drug\%20Review\%20Process/ pcodr_perc_deliberative_frame.pdf. Accessed November 28, 2018.

6. Masucci L, Beca J, Sabharwal M, et al. Methodological issues in economic evaluations submitted to the pan-Canadian Oncology Drug Review (pCODR). Pharmacoecon Open 2017;1:255-263.

7. Skedgel C, Wranik D, Hu M. The relative importance of clinical, economic, patient values and feasibility criteria in cancer drug reimbursement in Canada: a revealed preferences analysis of recommendations of the panCanadian Oncology Drug Review 2011-2017. Pharmacoeconomics 2018;36:467-475.

8. Hematology/Oncology (Cancer) Approvals \& Safety Notifications. U.S. Food \& Drug Administration website. Available at: https://www.fda.gov/ Drugs/InformationOnDrugs/ApprovedDrugs/ucm279174.htm. Accessed March 18, 2018

9. Cheema PK, Gavura S, Migus M, et al. International variability in the reimbursement of cancer drugs by publically funded drug programs. Curr Oncol 2012;19:e165-176.

10. Dusetzina SB. Drug pricing trends for orally administered anticancer medications reimbursed by commercial health plans, 2000-2014. JAMA Oncol 2016;2:960-961.

11. Dolgin E. Bringing down the cost of cancer treatment. Nature 2018;555:S26-29.

12. Smith TJ, Hillner BE. Bending the cost curve in cancer care. N Engl J Med 2011;364:2060-2065.
13. Aggarwal A, Fojo T, Chamberlain C, et al. Do patient access schemes for high-cost cancer drugs deliver value to society? Lessons from the NHS Cancer Drugs Fund. Ann Oncol 2017;28:1738-1750.

14. Allan GM, Lexchin J, Wiebe N. Physician awareness of drug cost: a systematic review. PLoS Med 2007;4:e283.

15. Wagner J, Marquart J, Ruby J, et al. Frequency and level of evidence used in recommendations by the National Comprehensive Cancer Network guidelines beyond approvals of the US Food and Drug Administration: retrospective observational study. BMJ 2018;360:k668.

16. DeMichele A. Present and future of CDK inhibitors. Presented at 2018 ASCO Annual Meeting; June 1-5, 2018; Chicago, Illinois.

17. $\mathrm{CADTH} / \mathrm{pCODR}$. Pan-Canadian Oncology Drug Review Initial Economic Guidance Report: Palbociclib (Ibrance) for Advanced Breast Cancer - Resubmission. Available at: https:/www.cadth.ca/sites/default/files/ pcodr/pcodr_palbociclib_ibrance_resub_abc_in_egr.pdf. Accessed August 7, 2018.

18. Niraula S, Amir E, Vera-Badillo F, et al. Risk of incremental toxicities and associated costs of new anticancer drugs: a meta-analysis. J Clin Oncol 2014;32:3634-3642.

19. Luce BR, Drummond $M$, Jonsson B, et al. EBM, HTA, and CER: clearing the confusion. Milbank Q 2010;88:256-276.

20. Mullard A. US drug spending hits $\$ 425$ billion. Nat Rev Drug Discov 2016;15:299

21. Mariotto AB, Yabroff KR, Shao $Y$, et al. Projections of the cost of cancer care in the United States: 2010-2020. J Natl Cancer Inst 2011;103:117128.

22. Burkholz H. The FDA Follies: An Alarming Look at our Food and Drugs in the 1980s. New York, NY: BasicBooks, HarperCollins Publishers; 1994.

23. Miller MT, Stromland K. Teratogen update: thalidomide: a review, with a focus on ocular findings and new potential uses. Teratology 1999;60:306321.

\section{See JNCCN.org for supplemental online content.}

